\title{
General interpolation by polynomial functions of distributive lattices
}

\author{
Miguel Couceiro ${ }^{1}$ Didier Dubois ${ }^{2}$ Henri Prade $^{2}$ Agnès Rico $^{3}$ Tamás \\ Waldhauser ${ }^{4,1}$ \\ 1 FSTC, University of Luxembourg, Luxembourg, miguel.couceiro@uni.lu \\ ${ }^{2}$ IRIT, CNRS and Université de Toulouse, France, \{dubois, prade\}@irit.fr \\ 3 ERIC, Université de Lyon, France, agnes.rico@univ-lyon1.fr \\ 4 Bolyai Institute, University of Szeged, Aradi vértanúk tere 1, H-6720 Szeged, \\ Hungary, twaldha@math.u-szeged.hu
}

\begin{abstract}
For a distributive lattice $L$, we consider the problem of interpolating functions $f: D \rightarrow L$ defined on a finite set $D \subseteq L^{n}$, by means of lattice polynomial functions of $L$. Two instances of this problem have already been solved.

In the case when $L$ is a distributive lattice with least and greatest elements 0 and 1 , Goodstein proved that a function $f:\{0,1\}^{n} \rightarrow L$ can be interpolated by a lattice polynomial function $p: L^{n} \rightarrow L$ if and only if $f$ is monotone; in this case, the interpolating polynomial $p$ was shown to be unique.

The interpolation problem was also considered in the more general setting where $L$ is a distributive lattice, not necessarily bounded, and where $D \subseteq L^{n}$ is allowed to range over cuboids $D=\left\{a_{1}, b_{1}\right\} \times \cdots \times\left\{a_{n}, b_{n}\right\}$ with $a_{i}, b_{i} \in L$ and $a_{i}<b_{i}$. In this case, the class of such partial functions that can be interpolated by lattice polynomial functions was completely described.

In this paper, we extend these results by completely characterizing the class of lattice functions that can be interpolated by polynomial functions on arbitrary finite subsets $D \subseteq L^{n}$. As in the latter setting, interpolating polynomials are not necessarily unique. We provide explicit descriptions of all possible lattice polynomial functions that interpolate these lattice functions, when such an interpolation is available.
\end{abstract}

\section{Introduction and Motivation}

The importance of aggregation functions is made apparent by their wide use, not only in pure mathematics (e.g., in the theory of functional equations, measure and integration theory), but also in several applied fields such as operations research, computer and information sciences, economics and social sciences, as well as in other experimental areas of physics and natural sciences. In particular, the use of aggregation functions has attracted much attention in decision theory for it provides an elegant and powerful formalism to model preference $[1,6]$ (for general background on aggregation functions, see [7]). 
In the qualitative approach to decision making, Sugeno integral $[14,15]$ remains one of the most noteworthy aggregation functions, and this is partially due to the fact that it provides a meaningful way to fuse or merge criteria within universes where essentially no structure, other than order, is assumed (unlike other classical aggregation functions, such as weighted arithmetic means, which require a rich arithmetical structure).

Even though primarily defined over real intervals, Sugeno integrals can be extended to wider domains (not only to arbitrary linearly ordered sets or chains, but also to bounded distributive lattices with bottom and top elements 0 and 1 , respectively) via the notion of lattice polynomial function. Essentially, a lattice polynomial function is a combination of variables and constants using the lattice operations $\wedge$ and $\vee$. As it turned out (see e.g. [2,9]), Sugeno integrals coincide exactly with those lattice polynomial functions that are idempotent (that is, which preserve constant tuples); in fact, it can be shown that the preservation of 0 and 1 suffices.

Apart from their theoretical interest, the consideration of Sugeno integrals on distributive lattices (rather than simply on scales or linearly ordered sets) or on finite Boolean algebras [10] is both useful and natural since scores for different criteria may be incomparable (for instance, when "ignorance" and "indifference" are possible scores).

Now in many real-life situations, we are faced with the problem of finding an aggregation operation based on scarce data that are not sufficient to determine a unique function. This fact gives rise to an interesting problem that appears in complete analogy with the classical theory of real polynomial functions, namely, that of interpolation. In this paper we are interested on aggregation functions defined over lattices and thus we consider the problem of interpolating lattice functions $f: L^{n} \rightarrow L$ on finite sets $D \subseteq L^{n}$ by means of Sugeno integrals or, more generally, by lattice polynomial functions.

The interpolation problem also has a learning flavor. The problem of covering a set of data by a set of Sugeno integrals, has been considered in the linearly ordered case [11,13]. More precisely, the condition of existence of a Sugeno integral interpolating a set of data has been laid bare, and the set of solutions (when non-empty) has been characterized as being upper and lower bounded by particular Sugeno integrals (easy to build from data). This process has been shown to be closely related to the version space approach in learning [12]. The theorems presented in this paper generalize these results to the more general case of partially ordered scales, and also open the possibility of the elicitation of families of generalized Sugeno integrals from, e.g., pieces of data where local and global evaluations may be imprecisely known.

The paper is organized as follows. In Section 2 we recall basic notions and terminology, provide the preliminary results needed throughout the paper, and address the interpolation problem we shall be dealing with. As it will become clear, a solution to this problem (when it exists) is not necessarily unique. Thus we present in Section 3 necessary and sufficient conditions for the existence of such a solution and, as a by-product, describe all possible solutions. Moreover, by 
confining ourselves to linearly ordered sets, we derive previous results obtained in this restricted setting. In Section 4 we address problems left open, and in Section 5 we discuss possible applications of our results.

\section{Preliminaries}

Throughout this paper let $L$ be a distributive lattice. Recall that a polynomial function over $L$ is a mapping $p: L^{n} \rightarrow L$ that can be expressed as a combination of the lattice operations $\wedge$ and $\vee$, projections and constants.

In the case when $L$ is bounded, i.e., with a least and a greatest element (which we denote by 0 and 1 , respectively), Goodstein [5] showed that polynomial functions $p: L^{n} \rightarrow L$ coincide exactly with those lattice functions that can be represented in disjunctive normal form (DNF for short) by

$$
p(\mathbf{x})=\bigvee_{I \subseteq[n]}\left(c_{I} \wedge \bigwedge_{i \in I} x_{i}\right), \text { where } \mathbf{x}=\left(x_{1}, \ldots, x_{n}\right) \in L^{n} \cdot{ }^{5}
$$

Furthermore, by taking $c_{I}^{*}:=\bigvee_{J \subseteq I} c_{J}$, we also have

$$
p(\mathbf{x})=\bigvee_{I \subseteq[n]}\left(c_{I}^{*} \wedge \bigwedge_{i \in I} x_{i}\right),
$$

and thus we can assume that the coefficients $c_{I}$ are monotone in the sense that $c_{I} \leq c_{J}$ whenever $I \subseteq J$. Under this monotonicity assumption we can recover the coefficients of the DNF from certain values of the polynomial function $p$. To this extent, for each $I \subseteq[n]$, let $\mathbf{1}_{I}$ be the tuple in $L^{n}$ whose $i$-th component is 1 if $i \in I$ and 0 if $i \notin I$. In this way, $p\left(\mathbf{1}_{I}\right)=c_{I}$.

These facts are reassembled in the following theorem, which asserts in particular that polynomial functions are uniquely determined by their restrictions to the hypercube $\{0,1\}^{n}$.

Theorem 1 (Goodstein [5]). Let $L$ be a bounded distributive lattice, and let $f$ be a function $f:\{0,1\}^{n} \rightarrow L$. There exists a polynomial function $p$ over $L$ such that $\left.p\right|_{\{0,1\}^{n}}=f$ if and only if $f$ is monotone. In this case $p$ is uniquely determined, and can be represented by the DNF

$$
p(\mathbf{x})=\bigvee_{I \subseteq[n]}\left(f\left(\mathbf{1}_{I}\right) \wedge \bigwedge_{i \in I} x_{i}\right) .
$$

Goodstein's theorem can be seen as a solution to an instance of the Interpolation Problem (see below), namely, interpolation on the hypercube $D=\{0,1\}^{n}$ : a function $f:\{0,1\}^{n} \rightarrow L$ can be interpolated by a polynomial function $p: L^{n} \rightarrow$ $L$ if and only if $f$ is monotone, and in this case $p$ is unique.

This result was generalized in [3] by allowing $L$ to be an arbitrary (possibly unbounded) distributive lattice and by considering functions $f: D \rightarrow L$, where

\footnotetext{
${ }^{5}$ As usual, the empty meet has value 1 and, dually, the empty join has value 0 .
} 
$D=\left\{a_{1}, b_{1}\right\} \times \cdots \times\left\{a_{n}, b_{n}\right\}$ with $a_{i}, b_{i} \in L$ and $a_{i}<b_{i}$, for each $i \in[n]$. Let $\widehat{\mathbf{e}}_{I}$ be the "characteristic vector" of $I \subseteq[n]$ (i.e., the tuple $\widehat{\mathbf{e}}_{I} \in L^{n}$ whose $i$-th component is $b_{i}$ if $i \in I$ and $a_{i}$ if $i \notin I$ ). The task of finding a polynomial function (or rather all polynomial functions) that takes prescribed values on the tuples $\widehat{\mathbf{e}}_{I}$ can be regarded as yet another instance of the Interpolation Problem.

Restricted Interpolation Problem Given $D:=\left\{\mathbf{e}_{I}^{D}: I \subseteq[n]\right\}$ and a function $f: D \rightarrow L$, find all polynomial functions $p: L^{n} \rightarrow L$ such that $\left.p\right|_{D}=f$.

In [3], necessary and sufficient conditions were established for the existence of an interpolating polynomial function. Moreover, it was shown that in this more general setting, uniqueness is not guaranteed, and all possible interpolating polynomial functions were provided, thus settling the Restricted Interpolation Problem.

In this paper we extend these results by solving the following general interpolation problem.

Interpolation Problem Let $L$ be a distributive lattice. Given an arbitrary finite set $D \subseteq L^{n}$ and $f: D \rightarrow L$, find all polynomial functions $p: L^{n} \rightarrow L$ such that $\left.p\right|_{D}=f$.

\section{Main results}

Let $D \subseteq L^{n}$ be a finite set, and consider a partial function $f: D \rightarrow L$. In this section we provide necessary and sufficient conditions that guarantee the existence of a polynomial function $p: L^{n} \rightarrow L$ that interpolates $f$, that is, $\left.p\right|_{D}=f$. As a by-product, we will determine all possible interpolating polynomial functions over a natural extension of the distributive lattice $L$ which we now describe.

By the Birkhoff-Priestley representation theorem, we can embed $L$ into a Boolean algebra $B$; see, e.g., [4]. For the sake of canonicity, we assume that $L$ generates $B$; under this assumption $B$ is uniquely determined up to isomorphism. The boundary elements of $B$ will be denoted by 0 and 1 . This notation will not lead to ambiguity since if $L$ has a least (resp. greatest) element, then it must coincide with 0 (resp. 1). The complement of an element $a \in B$ is denoted by $a^{\prime}: a \vee a^{\prime}=1$ and $a \wedge a^{\prime}=0$.

Given a function $f: D \rightarrow L$, we define the following two elements in $B$ for each $I \subseteq[n]$ :

$$
c_{I}^{-}:=\bigvee_{\mathbf{a} \in D}\left(f(\mathbf{a}) \wedge \bigwedge_{i \notin I} a_{i}^{\prime}\right) \quad \text { and } \quad c_{I}^{+}:=\bigwedge_{\mathbf{a} \in D}\left(f(\mathbf{a}) \vee \bigvee_{i \in I} a_{i}^{\prime}\right)
$$

Observe that $I \subseteq J$ implies $c_{I}^{-} \leq c_{J}^{-}$and $c_{I}^{+} \leq c_{J}^{+}$. Let $p^{-}$and $p^{+}$be the polynomial functions over $B$ given by these two systems of coefficients:

$$
p^{-}(\mathbf{x}):=\bigvee_{I \subseteq[n]}\left(c_{I}^{-} \wedge \bigwedge_{i \in I} x_{i}\right) \quad \text { and } \quad p^{+}(\mathbf{x}):=\bigvee_{I \subseteq[n]}\left(c_{I}^{+} \wedge \bigwedge_{i \in I} x_{i}\right)
$$


As it will become clear, $p^{-}$and $p^{+}$are the least and greatest polynomial functions over $B$ whose restriction to $D$ coincides with $f$ (whenever such a polynomial function exists). First, we need to establish a few auxiliary results.

Given $I \subseteq[n]$ and $\mathbf{a}=\left(a_{1}, \ldots, a_{n}\right), \mathbf{x}=\left(x_{1}, \ldots, x_{n}\right) \in B^{n}$, let $\mathbf{x}_{I}^{\mathbf{a}}$ be the $n$-tuple whose $i$-th component is $a_{i}$ if $i \in I$, and $x_{i}$ otherwise. If $I=\{i\}$, then we write $\mathbf{x}_{i}^{a_{i}}$ instead of $\mathbf{x}_{I}^{\mathbf{a}}$.

Lemma 1. Let $p: B^{n} \rightarrow B$ be a polynomial function over $B$. For $I \subseteq[n]$ and $\mathbf{x} \in B^{n}$, the polynomial functions $q_{I}^{-}$and $q_{I}^{+}$given by

$$
q_{I}^{-}(\mathbf{a}):=p\left(\mathbf{x}_{I}^{\mathbf{a}}\right) \wedge \bigwedge_{i \in I} a_{i}^{\prime} \quad \text { and } \quad q_{I}^{+}(\mathbf{a}):=p\left(\mathbf{x}_{I}^{\mathbf{a}}\right) \vee \bigvee_{i \in I} a_{i}^{\prime},
$$

are nonincreasing.

Proof. Since monotonicity is defined componentwise, to show that $q_{I}^{-}$is nonincreasing it suffices to consider the case when $I$ is a singleton.

So let $I=\{i\}$ and $\mathbf{a} \in B^{n}$. In this case, $p\left(\mathbf{x}_{I}^{\mathbf{a}}\right)$ can be regarded as a unary polynomial function and thus, by (1), it is of the form $s \vee\left(a_{i} \wedge t\right)$, for some $s \leq t$. Hence, $q_{I}^{-}$can be expressed as

$$
q_{I}^{-}(\mathbf{a})=\left(s \vee\left(a_{i} \wedge t\right)\right) \wedge a_{i}^{\prime} .
$$

By distributivity, we get $q_{I}^{-}(\mathbf{a})=s \wedge a_{i}^{\prime}$, which shows that the first claim holds. The second claim follows dually, and the proof of the lemma is complete.

Corollary 1. Let $p: B^{n} \rightarrow B$ be a polynomial function over $B$. For every $I \subseteq$ $[n]$ and $\mathbf{a} \in B^{n}$,

$$
p\left(\mathbf{1}_{I}\right) \leq p(\mathbf{a}) \vee \bigvee_{i \in I} a_{i}^{\prime} \quad \text { and } \quad p\left(\mathbf{1}_{I}\right) \geq p(\mathbf{a}) \wedge \bigwedge_{i \notin I} a_{i}^{\prime}
$$

Proof. Let $\mathbf{b}=\mathbf{a}_{[n] \backslash I}^{0}$; clearly, $\mathbf{a}, \mathbf{1}_{I} \geq \mathbf{b}$. Since $p$ is nondecreasing, we have $p\left(\mathbf{1}_{I}\right) \geq p(\mathbf{b})$, and since $b_{i}^{\prime}=1$ for every $i \in[n] \backslash I$, it follows from Lemma 1 that

$$
p\left(\mathbf{1}_{I}\right) \geq p(\mathbf{b}) \wedge \bigwedge_{i \notin I} b_{i}^{\prime} \geq p(\mathbf{a}) \wedge \bigwedge_{i \notin I} a_{i}^{\prime} .
$$

This shows that the second claim holds; the first follows similarly.

Using Corollary 1, we can now provide necessary conditions on the coefficients of interpolating polynomials.

Lemma 2. Let $f: D \rightarrow L$ be a function defined on a finite set $D \subseteq L^{n}$, and let $p: B^{n} \rightarrow B$ be a polynomial function over $B$ given by (1). If $\left.p\right|_{D}=f$, then $c_{I}^{-} \leq c_{I} \leq c_{I}^{+}$. 
Proof. Suppose $\left.p\right|_{D}=f$. By Corollary 1 , for every $I \subseteq[n]$ and $\mathbf{a} \in D$,

$$
p(\mathbf{a}) \wedge \bigwedge_{i \notin I} a_{i}^{\prime} \leq p\left(\mathbf{1}_{I}\right) \leq p(\mathbf{a}) \vee \bigvee_{i \in I} a_{i}^{\prime} .
$$

Since $p(\mathbf{a})=f(\mathbf{a})$, for every $\mathbf{a} \in D$, it follows that

$$
\bigvee_{\mathbf{a} \in D}\left(f(\mathbf{a}) \wedge \bigwedge_{i \notin I} a_{i}^{\prime}\right) \leq p\left(\mathbf{1}_{I}\right) \leq \bigwedge_{\mathbf{a} \in D}\left(f(\mathbf{a}) \vee \bigvee_{i \in I} a_{i}^{\prime}\right),
$$

and hence $c_{I}^{-} \leq c_{I} \leq c_{I}^{+}$as desired.

To show that this condition is also sufficient, we make use of the following lemma.

Lemma 3. Let $f: D \rightarrow L$ be a function defined on a finite set $D \subseteq L^{n}$. Then $p^{-}(\mathbf{b}) \geq f(\mathbf{b}) \geq p^{+}(\mathbf{b})$, for every $\mathbf{b} \in D$.

Proof. By definition of $p^{-}$and using the fact that for each $I \subseteq[n]$,

$$
c_{I}^{-}=\bigvee_{\mathbf{a} \in D}\left(f(\mathbf{a}) \wedge \bigwedge_{i \notin I} a_{i}^{\prime}\right) \geq f(\mathbf{b}) \wedge \bigwedge_{i \notin I} b_{i}^{\prime},
$$

we have

$$
\begin{aligned}
p^{-}(\mathbf{b}) & =\bigvee_{I \subseteq[n]}\left(c_{I}^{-} \wedge \bigwedge_{i \in I} b_{i}\right) \geq \bigvee_{I \subseteq[n]}\left(f(\mathbf{b}) \wedge \bigwedge_{i \notin I} b_{i}^{\prime} \wedge \bigwedge_{i \in I} b_{i}\right) \\
& =f(\mathbf{b}) \wedge \bigvee_{I \subseteq[n]}\left(\bigwedge_{i \notin I} b_{i}^{\prime} \wedge \bigwedge_{i \in I} b_{i}\right) .
\end{aligned}
$$

By repeated application of distributivity, it is not difficult to verify that

$$
\bigvee_{I \subseteq[n]}\left(\bigwedge_{i \notin I} b_{i}^{\prime} \wedge \bigwedge_{i \in I} b_{i}\right)=1,
$$

and thus $p^{-}(\mathbf{b}) \geq f(\mathbf{b})$. Dually, we can also verify that $f(\mathbf{b}) \geq p^{+}(\mathbf{b})$, which completes the proof.

We can now characterize the class of functions over a distributive lattice $L$ that can be interpolated on finite domains by polynomial functions over the Boolean algebra generated by $L$. In fact, the following theorem explicitly determines all possible interpolating polynomial functions, whenever such polynomial functions exist.

Theorem 2. Let $f: D \rightarrow L$ be a function defined on a finite set $D \subseteq L^{n}$, and let $p: B^{n} \rightarrow B$ be a polynomial function over $B$ given by (1). Then the following conditions are equivalent:

1. $p$ interpolates $f$, i.e., $\left.p\right|_{D}=f$; 
2. $c_{I}^{-} \leq c_{I} \leq c_{I}^{+}$;

3. $p^{-} \leq p \leq p^{+}$.

Proof. Clearly, $(2) \Longleftrightarrow(3)$. By Lemma $2,(1) \Longrightarrow(2)$. Moreover, if (3) holds, then using Lemma 3 we conclude that for every $\mathbf{b} \in D$,

$$
f(\mathbf{b}) \leq p^{-}(\mathbf{b}) \leq p(\mathbf{b}) \leq p^{+}(\mathbf{b}) \leq f(\mathbf{b}) .
$$

Hence, $\left.p\right|_{D}=f$.

From Theorem 2 it follows that a necessary and sufficient condition for the existence of a polynomial function $p: B^{n} \rightarrow B$ such that $\left.p\right|_{D}=f$ is $c_{I}^{-} \leq c_{I}^{+}$, for every $I \subseteq[n]$.

Moreover, if for every $I \subseteq[n]$, there is $c_{I} \in L$ such that $c_{I}^{-} \leq c_{I} \leq c_{I}^{+}$, then and only then there is a polynomial function $p: L^{n} \rightarrow L$ such that $\left.p\right|_{D}=f$.

The latter condition leads us to considering the case when $L$ is a complete lattice (i.e., for which arbitrary joins and meets exist), and to defining two operators $c l$ and int as follows: For $b \in B$, let

$$
\operatorname{cl}(b):=\bigwedge_{x \in L x \geq b} x \text { and } \operatorname{int}(b):=\bigvee_{x \in L x \leq b} x
$$

It is not difficult to see that for any $b_{1}, b_{2} \in B$, we have

$$
\operatorname{cl}\left(b_{1} \vee b_{2}\right)=\operatorname{cl}\left(b_{1}\right) \vee c l\left(b_{2}\right) \quad \text { and } \quad \operatorname{int}\left(b_{1} \wedge b_{2}\right)=\operatorname{int}\left(b_{1}\right) \wedge \operatorname{int}\left(b_{2}\right) .
$$

Using this fact, the necessary and sufficient condition given above for the existence of an interpolating polynomial function over $L$ can be translated into the following system of inequalities.

Corollary 2. If $L$ is complete, then there is a polynomial function $p: L^{n} \rightarrow L$ such that $\left.p\right|_{D}=f$ if and only if for every $I \subseteq[n]$ there is $c_{I} \in L$ such that $c_{I}^{-} \leq c_{I} \leq c_{I}^{+}$or, equivalently,

$$
\operatorname{cl}\left(c_{I}^{-}\right)=\bigvee_{\mathbf{a} \in D} \operatorname{cl}\left(f(\mathbf{a}) \wedge \bigwedge_{i \notin I} a_{i}^{\prime}\right) \leq \bigwedge_{\mathbf{a} \in D} \operatorname{int}\left(f(\mathbf{a}) \vee \bigvee_{i \in I} a_{i}^{\prime}\right)=\operatorname{int}\left(c_{I}^{+}\right),
$$

for every $I \subseteq[n]$.

Remark 1. Corollary 2 generalizes Theorem 10 in [3] which established necessary and sufficient conditions for the existence of a solution of the Restricted Interpolation Problem.

In the case when $L$ is a finite chain, a solution to the Interpolation Problem was given in [11] and where, rather than polynomial functions, the interpolating functions were assumed to be Sugeno integrals, i.e., idempotent polynomial functions; see $[8,9]$.

Now if $L$ is a finite chain, then

$$
\begin{aligned}
& c l\left(c_{I}^{-}\right)=\bigvee\left\{f(\mathbf{a}): \mathbf{a} \in D, \quad \forall i \notin I, \quad a_{i}<f(\mathbf{a})\right\}, \quad \text { and } \\
& \operatorname{int}\left(c_{I}^{+}\right)=\bigwedge\left\{f(\mathbf{a}): \mathbf{a} \in D, \quad \forall i \in I, \quad a_{i}>f(\mathbf{a})\right\} .
\end{aligned}
$$


Hence, $\operatorname{cl}\left(c_{I}^{-}\right) \leq \operatorname{int}\left(c_{I}^{+}\right)$if and only if for every $\mathbf{a}, \mathbf{b} \in D$,

$$
f(\mathbf{a})>f(\mathbf{b}) \Rightarrow \exists i \notin I: a_{i} \geq f(\mathbf{a}) \quad \text { or } \quad \exists i \in I: b_{i} \leq f(\mathbf{b}) .
$$

It is not difficult to see that condition (2) holds for every $I \subseteq[n]$ if and only if condition (3) (see theorem below) also holds. The following result basically reformulates Theorem 3 of [11] in the language of lattice theory.

Theorem 3 ([11]). Let $L$ be a finite chain, and let $f: D \rightarrow L$ be a function defined on a finite subset $D \subseteq L^{n}$. Then there exists a polynomial function $p: L^{n} \rightarrow L$ such that $\left.p\right|_{D}=f$ if and only if

$$
\forall \mathbf{a}, \mathbf{b} \in D: f(\mathbf{a})>f(\mathbf{b}) \Rightarrow \exists i \in[n]: b_{i} \leq f(\mathbf{b})<f(\mathbf{a}) \leq a_{i} .
$$

\section{Further work}

When we consider a function $f: D \rightarrow L$, a polynomial function can be indentified if and only if for every $I \subseteq[n]$ there exists $c_{I} \in L$ such that $c_{I}^{-} \leq c_{I} \leq c_{I}^{+}$. In some practical cases this condition would be not satisfied, i.e., the data would be inconsistent with respect to a polynomial function. In such a case one issue can be to build a family of polynomial functions as it is done in the totally ordered context in [13]. More precisely, a partition of the dataset can be built in which each set of the partition is compatible with a family of polynomial functions. In general, many partitions are possible since their definition without other conditions depends on the order in which the data are considered. We can add a condition to choose one partition. For example, we can decide to look for the smallest partition in terms of number of subsets.

The complexity to check the conditions $c_{I}^{-} \leq c_{I}^{+}$for all $I \subseteq[n]$ is at least $2^{n}$, so these conditions become costly when $n$ increases. In the totally ordered context presented in [11] the complexity can be reduced since the check only needs $p^{2}$ comparisons of compatibility where $p$ is the cardinality of $D$. This raises the question of finding a similar result in our context.

\section{Applications and concluding remarks}

To replace missing data is a common problem for the statistical treatment of data. There are many methods to give a value to the missing data. On the other hand, Sugeno integral is classically used in decision theory to represent the behavior of a decision-maker. Since polynomials functions are a generalization of Sugeno integrals, it seems natural to make use of these polynomial functions to replace the missing data.

Acknowledgments. The first named author is supported by the internal research project F1R-MTH-PUL-09MRDO of the University of Luxembourg. The fifth named author acknowledges that the present project is supported by the 
TÁMOP-4.2.1/B-09/1/KONV-2010-0005 program of the National Development Agency of Hungary, by the Hungarian National Foundation for Scientific Research under grants no. K77409 and K83219, by the National Research Fund of Luxembourg, and cofunded under the Marie Curie Actions of the European Commission (FP7-COFUND).

\section{References}

1. Bouyssou, D., Dubois, D., Prade, H., Pirlot, M. (eds): Decision-Making Process Concepts and Methods. ISTE/John Wiley (2009)

2. Couceiro, M., Marichal, J.-L.: Characterizations of discrete Sugeno integrals as polynomial functions over distributive lattices. Fuzzy Sets and Systems 161(5), 694-707 (2010)

3. Couceiro, M., Waldhauser, T.: A generalization of Goodstein's theorem: interpolation by polynomial functions of distributive lattices. http://arxiv .org/abs/1110. 0321

4. Davey, B. A., Priestley, H. A.: Introduction to Lattices and Order. Cambridge University Press, New York (2002)

5. Goodstein, R. L.: The Solution of Equations in a Lattice. Proc. Roy. Soc. Edinburgh Section A, 67, 231-242 (1965/1967)

6. Grabisch, M.: The application of fuzzy integrals in multicriteria decision making. Eur. J. Oper. Res. 89:3, 445-456 (1996)

7. Grabisch, M., Marichal, J.-L., Mesiar, R., Pap, E.: Aggregation Functions. Encyclopedia of Mathematics and its Applications. Cambridge University Press, Cambridge (2009)

8. Marichal, J.-L.: On Sugeno integral as an aggregation function. Fuzzy Sets and Systems 114, 347-365 (2000).

9. Marichal, J.-L.: Weighted lattice polynomials. Discrete Mathematics 309(4), 814$820(2009)$

10. Rico, A: Sugeno integral in a finite Boolean algebra. Fuzzy Sets and Systems, 159, 1709-1718 (2008)

11. Rico, A., Grabisch, M., Labreuche, Ch., Chateauneuf, A.: Preference modeling on totally ordered sets by the Sugeno integral. Discrete Applied Math. 147(1), 113-124 (2005)

12. Prade, H., Rico, A., Serrurier, M. Elicitation of Sugeno Integrals: A version space learning perspective. Proc. 18th Inter. Symp. on Methodologies for Intelligent Systems (ISMIS'09), (J. Rauch, Z. W. Ras, P. Berka, T. Elomaa, eds.), Prague, Sept. 14-17, Springer, LNCS 5722, 392-401 (2009).

13. Prade, H., Rico, A., Serrurier, M., Raufaste E.: Eliciting Sugeno integrals: Methodology and a case study, 2009, in Proc. European Conf. on Symbolic and Quantitative Approaches to Reasoning with Uncertainty, ECSQARU'09, LNCS, pages: $712-723$.

14. Sugeno, M.: Theory of Fuzzy Integrals and its Applications. PhD thesis, Tokyo Institute of Technology, Tokyo (1974)

15. Sugeno, M.: Fuzzy measures and fuzzy integrals - a survey. In: Gupta, M. M., Saridis, G. N., Gaines, B. R., (eds), Fuzzy automata and decision processes, pp. 89-102. North-Holland, New York (1977) 